syndrome (IBS) and associated consumption of healthcare resources in the Scottish National Health Service.

Methods A consultation records database covering 56 general practitioner (GP) practices in Scotland (approximately 255,000 people; $5.8 \%$ of the population) was used to identify consultations primarily due to IBS with GP or practice nurses between April 2009 and March 2011 (Read codes: IBS, constipation, diarrhoea). Read codes suggesting other causes of diarrhoea or constipation and patients with inflammatory bowel disease, diverticular disease, coeliac disease or bowel cancer were excluded. A national primary care prescription database was used to identify use of antispasmodics, laxatives or antidiarrhoeals. Referrals to outpatient clinics and acute hospital admissions between January and December 2011 in antispasmodictreated patients were also analysed using national datasets.

Results Based on consultation records, an estimated 341,180 adults ( $\geq 18$ years) in Scotland suffer from IBS, representing an estimated prevalence of $7.7 \%$ (females: 9.8\%; males: $5.5 \%$ ). Patients consulted 1.05 times during 2010/11 for IBS symptoms and consulted more frequently overall than the general population (9.3 vs. 4.7 times per annum).

During 2011, 142,738 adults received $\geq 1$ prescription for antispasmodics, most commonly mebeverine (40.1\%), hysocine butylbromide $(35.7 \%)$ or peppermint $(18.0 \%)$, giving an estimated prevalence of antispasmodic-treated IBS of 3.4\% (females: 4.7\% males: $2.0 \%$ ). One-third of these patients were also prescribed laxatives (24.5\%), the antidiarrhoeal loperamide (6.7\%) or both $(2.5 \%)$. Of the antispasmodic-treated patients, 11,645 $(9.0 \%)$ visited a gastroenterology outpatient clinic in 2011 $(11.7 \%$ of all gastroenterology clinic attendances) and 1,869 (1.3\%) were acutely admitted to hospital due to IBS or symptoms likely to be associated with IBS, most frequently constipation (80.3\%). The average length of hospital stay was 2.1 days (2.4 days for admissions due to constipation). A further 1,141 people with no record of antispasmodic prescriptions were admitted with a primary diagnosis of IBS.

Conclusion Based on consultation records, the estimated prevalence of IBS in Scotland is 7.7\%, and 3.4\% based on prescription records; these estimates exclude patients who do not consult a GP or who use laxatives alone or over-the-counter medicines. The prevalence estimates and levels of treatment in this study were consistent with other published information, and show that patients with IBS consume significant healthcare resources.

Study commissioned by Almirall UK.

Disclosure of Interest S. McTaggart Grant/research support from: This study was undertaken as a commercially contracted piece of work for Almirall UK, G. Wyper Grant/research support from: This study was undertaken as a commercially contracted piece of work for Almirall UK, L. Harkins Grant/research support from: This study was undertaken as a commercially contracted piece of work for Almirall UK, I. Bishop Grant/research support from: This study was undertaken as a commercially contracted piece of work for Almirall UK, M. Bennie Grant/research support from: This study was undertaken as a commercially contracted piece of work for Almirall UK, M. Rance Employee of: Almirall.

\section{PWE-177 A SYSTEMATIC REVIEW OF ANTIDEPRESSANTS IN IRRITABLE BOWEL SYNDROME: A QUALITATIVE ANALYSIS}

${ }^{1} \mathrm{M}$ Rance ${ }^{*},{ }^{2} \mathrm{~L}$ Lindner, ${ }^{3} \mathrm{AC}$ Ford. ${ }^{1}$ Almirall UK, Uxbridge, UK, ${ }^{2}$ Almirall S. A., Barcelona, Spain; ' Leeds University and Leeds Teaching Hospitals Trust, Leeds, UK

10.1136/gutjnl-2014-307263.437
Introduction Antidepressants have a mild analgesic effect and are commonly used in the management of irritable bowel syndrome (IBS); however, the quality of the clinical evidence to support the use of tricyclic antidepressants (TCAs) and selective serotonin inhibitors (SSRIs) in IBS is unclear. The aim of this literature review was to systematically identify and analyse published evidence on the efficacy of TCAs and SSRIs for IBS.

Methods A systematic search of the medical literature was conducted using the PubMed, Embase, and Cochrane databases. Search terms included 'irritable bowel syndrome', 'spastic colon', 'irritable colon', 'functional diseases, colon' and a mixture of agent terms - including antidepressants, tricyclic, and SSRIs. Randomised placebo-controlled trials evaluating the efficacy of antidepressants (SSRIs and TCAs) in adult patients with IBS were eligible for inclusion. Exclusion criteria included absence of placebo arm, patients $<18$ years of age, and dual publication. Only antidepressants available in the UK were included in this analysis.

Results A total of 628 unique titles and abstracts were retrieved; 581 records were excluded upon title or abstract review and 33 upon full-text review. The final review included 14 studies: 7 reporting on TCAs, 6 on SSRIs, and 1 comparing both an SSRI and a TCA vs. placebo. Agents included in the analysis were citalopram, paroxetine, fluoxetine, imipramine, amitriptyline, trimipramine and doxepin. Overall, 797 patients were included in the 14 studies, with 240 receiving a TCA, 171 receiving an SSRI and 386 receiving placebo. Of the nine studies (475 patients) that reported IBS subtype, $50 \%$ of patients had diarrhoea-predominant IBS, $32 \%$ had constipation-predominant IBS (IBS-C) and 19\% had alternating-type IBS. Treatment duration ranged from 4-12 weeks. The most common IBS-related outcome measures were for global symptom relief and improvements in abdominal pain/discomfort. Three studies reported on quality of life, while only limited data were available for treatment satisfaction. Most outcomes did not align well with those now required for FDA and EMA regulatory approval of medicines to treat IBS-C.

Conclusion The evidence base for the use of antidepressants in IBS is of low quality, making estimates of effect very uncertain. Data for the efficacy of antidepressants in IBS subtypes are especially limited. Further studies are required to support the offlabel use of antidepressants in IBS.

Study funded by Almirall UK.

Disclosure of Interest M. Rance Employee of: Almirall UK, L. Lindner Employee of: Almirall S. A., A. C. Ford: None Declared.

\section{PWE-178 IMPACT OF CHRONIC CONSTIPATION ON HEALTHCARE RESOURCE USE IN THE UK: AN ANALYSIS BASED ON ELECTRONIC MEDICAL RECORDS}

${ }^{1} \mathrm{M}$ Radford*, ${ }^{1} \mathrm{E}$ Bloomfield, ${ }^{2} \mathrm{~A}$ Joseph. 'IMS Health, London, UK; ${ }^{2}$ Shire, Eysins, Switzerland

\subsection{6/gutjnl-2014-307263.438}

Introduction Chronic constipation is a common condition with a substantial impact on health-related quality of life. The aim of this study was to assess healthcare resource use and prescription laxative costs associated with chronic constipation in the UK, using electronic medical records.

Methods The IMS Disease Analyzer database contains electronic medical records from primary healthcare practices in England and Wales. Patients were identified as having chronic constipation if they had: $\geq 2$ international classification of diseases-revision 10 (ICD-10) codes for constipation within 12 months; or 1 primary constipation diagnosis and $\geq 2$ laxative prescriptions in the 6 months before or 12 months after the constipation 
diagnosis. Patients with a diagnosis of irritable bowel syndrome or a record of opioid use were excluded. Records were analysed from 1 January 2008 to 31 December 2011 for the 12-month period following the first constipation diagnosis. Mortality, frequency of comorbidities, number of healthcare consultations, and total laxative prescriptions and costs were recorded and stratified by sex and age group (age 18-49, 50-64, 65-74, and $\geq 75$ years). For patients with less than 12 months follow-up, data on costs, healthcare contacts and prescriptions were adjusted to an annual rate using a simple linear projection. Regression analyses were performed on a case basis to estimate the impact of covariates on laxative costs.

Results Overall, 10371 patients with chronic constipation were identified, of whom $63 \%$ were women and $53 \%$ were $\geq 75$ years old. Mortality during the 12 -month follow up period was $8.4 \%$. The most common co-morbidities were primary hypertension (32\%) and type 2 diabetes mellitus (13\%). Patients had a mean of 27.7 consultations (standard deviation [SD] 24.0), including 4.0 (SD 9.8) for constipation-related care, in the 12-month follow up period. In total, $92 \%$ of patients were prescribed laxatives; patients had on average 8.3 (SD 14.0) laxative prescriptions in the 12 month follow up, which using an average prescription cost of $£ 4.28$ resulted in a mean cost to the National Health Service of $£ 35.41 /$ person/year in 2011 GBP. An increase in prescription laxative cost was significantly associated with increasing age group, the presence of Parkinson's disease (proportional cost increase [PCI] $1.29, \mathrm{p}<0.0005)$, type 2 diabetes mellitus (PCI 1.08, $\mathrm{p}<0.025$ ) and multiple sclerosis (PCI 1.51, $\mathrm{p}<0.0005$ ).

Conclusion In this analysis of UK electronic medical records using the IMS UK Disease Analyzer database, the majority of patients with chronic constipation were elderly, and over $90 \%$ were prescribed laxatives. Higher treatment costs were associated with increased age and co-morbidity.

Disclosure of Interest M. Radford Employee of: IMS Health, which received funding from Shire to carry out this research., E. Bloomfield Employee of: IMS Health, which received funding from Shire to carry out this research., A. Joseph Employee of: Shire.

\section{PWE-179 A SURVEY ON PATIENT WELLBEING AND PATIENT COMFORT DURING OESOPHAGEAL INTUBATIONS. A MULTICENTRE STUDY}

${ }^{1} \mathrm{M}$ Brennan*, ${ }^{*} \mathrm{~T}$ Moran, ${ }^{1} \mathrm{P}$ Lawlor, ${ }^{2} \mathrm{~L}$ Barry, ${ }^{3} \mathrm{M}$ Treacy, ${ }^{4} \mathrm{JV}$ Reynolds, ${ }^{4} \mathrm{R}$ Narayanasamy. ${ }^{1}$ Gl Function Unit, St James Hospital, Dublin 8, Ireland; ${ }^{2}$ Clinical Measurement Lab, Mercy University Hospital, Cork, Ireland; ' University College Hospital, Galway, Co. Galway, Ireland; ${ }^{4}$ Department of Surgery, St James Hospital, Dublin 8, Ireland

10.1136/gutjnl-2014-307263.439
Introduction A Multicentre survey was undertaken from November 2013 until January 2014 on 80 patients (45f Vs $35 \mathrm{~m}$ ) undergoing oesophageal manometry and/or oesophageal $24 \mathrm{hr} \mathrm{pH} /$ impedance study. The aim of this study was to determine the overall wellbeing of the patient during their investigation. Oesophageal intubations are invasive techniques which many patients find quite distressing. These studies require the patient to be alert and to be able to swallow liquid boluses. In order to retain their normal oesophageal function, sedation or oral anaesthetic spray is not routinely administered. This study was compiled to obtain specific patient data from Gastrointestinal (GI) Units throughout Ireland by conducting a survey post patient procedure.

Methods A survey containing short answer questions was devised and GI units providing a service in GI Physiology testing were asked to participate. The patient was asked to answer either 'Yes' or 'No' to the questions or score the answers to the questions with a value from $0-10$ with 10 being the most severe scale of discomfort/anxiety and 0 being the least.

Results Table 1 below shows the results obtained from the multicentre survey.

The reasons given for failed intubations included patient anxiety, nasal sensitivity and previous traumatic Endoscopy experience. With regards to patient anxiety prior to investigation; no option for general anaesthetic, sedation, nasal spray, throat spray, and the fear of the unknown were the main reasons for the high patient anxiety scores.

Conclusion Appropriately trained GI Physiologists achieved a 96.3\% rate of successful oesophageal intubations. Despite this, patient anxiety in anticipation of their procedure is relatively high. With the option of a nasal spray, this survey suggests that patient anxiety levels prior to their investigation would be reduced, thus making the intubation a more pleasant, tolerable and less traumatic experience.

Disclosure of Interest None Declared.

\section{PWE-180 A SURVEY EVALUATING GENERAL PRACTITIONERS', GASTROENTEROLOGISTS' AND EXPERTS' DIAGNOSTIC APPROACHES TO INFLAMMATORY BOWEL DISEASE, IRRITABLE BOWEL SYNDROME AND CHRONIC CONSTIPATION IN FIVE EUROPEAN COUNTRIES}

${ }^{1} V$ Andresen, ${ }^{2} \mathrm{P}$ Whorwell*, ${ }^{3} \mathrm{~J}$ Fortea, ${ }^{4} \mathrm{~J}$ Milce. ${ }^{1} /$ sraelitisches Krankenhaus, Hamburg, Germany; ${ }^{2}$ Wythenshawe Hospital, Manchester, UK; ${ }^{3}$ Almirall S. A., Barcelona, Spain; ${ }^{4}$ Kantar Health, Paris, France

10.1136/gutjnl-2014-307263.440

\begin{tabular}{|c|c|c|c|}
\hline & Total & Female & Male \\
\hline Mean Age (years) & 49.23 & 53.58 & 43.63 \\
\hline Successful intubation rate\% & $96.3 \%$ & $95.6 \%$ & $97.1 \%$ \\
\hline Successful intubation rate in patients & $94.4 \%$ & $94.4 \%$ & $94.4 \%$ \\
\hline \multicolumn{4}{|l|}{$>40$ years } \\
\hline Successful intubation rate in patients & $100 \%$ & $100 \%$ & $100 \%$ \\
\hline \multicolumn{4}{|l|}{$<40$ years } \\
\hline Mean anxiety score prior to investigation * & 4.73 & 5.47 & 3.77 \\
\hline Mean anxiety score post procedure * & 1.96 & 2.26 & 1.59 \\
\hline Mean discomfort level & 4.91 & 4.93 & 4.89 \\
\hline Number of patients who would be anxious if studies had to be repeated & 32.55 & $35.56 \%$ & $28.57 \%$ \\
\hline Number of patients who would choose to have an anaesthetic spray administered & $61.25 \%$ & $66.67 \%$ & $54.29 \%$ \\
\hline
\end{tabular}

